\title{
REPRESENTATIONS OF POLYCRYSTALLINE MICROSTRUCTURE BY $n$-POINT CORRELATION TENSORS
}

\author{
PAVEL ETINGOF $\dagger$ and BRENT L. ADAMS $\ddagger$ \\ Departments of Mathematics $\dagger$ and Mechanical Engineering, $¥$ Yale University, \\ New Haven, CT, 06520
}

(Received 6 June, 1992)

\begin{abstract}
An important class of representations of polycrystalline microstructure consists of the $n$-point correlation tensors. In this paper the representation theory of groups is applied to a consideration of symmetries in the $n$-point correlation tensors. Three sources of symmetry are included in the development: indicial symmetry in the coefficients of tensors, symmetry associated with the crystal lattice, and statistical symmetries in the microstructure induced by processing. The central problem discussed here is the "residence space", or the space of minimum dimension occupied by correlation tensors possessing such symmetries. In addition to the general case of correlation tensors possessing such symmetries, a model microstructure is also considered which embodies an assumption of no spatial coherence of lattice orientation between neighboring grains or crystallites. It is shown that the model microstructure generally results in residence spaces of lower dimension.
\end{abstract}

KEY WORDS Polycrystalline microstructure, $n$-point correlation tensors, residence space, symmetries.

\section{INTRODUCTION}

Microstructure refers to any of the myriad of features observed by the experimentalist when probing the internal arrangement of components of solid materials. In polycrystals microstructure refers to aspects of its constituent grains, including the distribution and spatial correlation of material points by chemical phase and lattice orientation, the size and shape distribution of the crystallites, their topological connectivity, and the density and distribution of lattice defects. Observations of microstructure are always limited by probing instruments to a restricted set of accessible features. Experiments yield transformed or projected views of the true microstructure since probing is always limited in resolution (spatial, wavelength, etc.). A consequence of the fact that most solids are opaque is a recourse to certain principles of stereology in order to infer information about the three-dimensional microstructure from two-dimensional plane sectioning. Even if the observer had an unobscured access to the microstructure, he is limited in understanding the relevance of microstructural observations to particular properties and behavior of interest. Deep physical insight into the associations between microstructural features and behavior is required to understand their importance. 
It is in this complicated setting that we shall consider a particular and specialized class of representations of polycrystalline microstructure that frequently appears in property bounding theories. $N$-point correlation tensors naturally occur in estimates and bounding theories of linear properties (cf. Beran and Molyneux, 1966; Hashin and Shtrikman, 1962; Kröner, 1986; McCoy, 1981; Willis, 1981). More recently the same class of representations has been shown to occur in some nonlinear estimates and bounds (cf. Willis 1983, Talbot and Willis 1985, Ponte-Casteñada 1991, Adams and Field 1991). It would appear that representation by $n$-point tensors has significant breadth of application in materials science. The goal of the paper is to carefully consider three classes of symmetry which appear in such representations: the indicial symmetry of selected material tensors, symmetries which arise in the crystal lattice, and symmetries arising during thermomechanical processing of the polycrystal.

Representation of microstructure refers to any mathematical classification of features present in microstructure. Such representation is always limited in scope. Complete representation of microstructure in polycrystalline materials is neither possible nor is it desirable. Rather, we must seek to find a minimal representation of microstructure which correlates, to the desired resolution, with those properties and behaviors of interest. It is in this context that consideration of symmetry becomes an important factor, since symmetry analysis helps identify the minimal required representation. More precisely, the goal is to define the smallest space of representation of the microstructure (consistent with the indicial, lattice and processing symmetries present in the material) and the related minimum set of experimental measurements required to construct the representation.

In the sections which follow we first define the $n$-point correlation tensors in the context of materials science and the modern quantitative texture theory of microstructure. Subsequently, a section is furnished containing a few results of the representation theory of finite groups and compact Lie groups. We make no attempt to derive these results; our primary goal is to establish some definitions and vocabulary that will be required in the subsequent development. Readers not familiar with representation theory of groups will find an extensive published literature (cf. Gel'fand, Minlos and Shapiro 1963; Broecker and tom Dieck 1985). The main section of the paper describes a general approach to symmetry considerations in the $\boldsymbol{n}$-point tensors. Prescriptions for application of the general approach to specific cases is somewhat varied, and we shall not attempt to describe it here. Rather, we elect to give results for a selected set of problems ranging from the most elementary to examples which have substantial technological importance.

\section{REPRESENTATION OF MICROSTRUCTURE BY $\boldsymbol{n}$-POINT CORRELATION TENSORS}

Some fundamental aspects of microstructure in polycrystalline materials can be described in terms of an ideal reference crystal. This reference crystal is modeled as the repetition of a particular structural basis upon a reference lattice of points in $\mathbb{R}^{3}$. The lattice of points is defined by the vectors $\mathbf{v}=v^{i} \mathbf{a}_{i}$ where $\left\{\mathbf{a}_{i}\right\}$ is the set 
of basic vectors of the lattice and $v^{i} \in \mathbb{Z}$, the set of integers. For the moment we shall consider that no particular point symmetry is present in the lattice.

Consider affine transformations of the ideal reference crystal. Such transformations alter the lattice of points of the reference crystal according to the following formula:

$$
\mathbf{v} \rightarrow \tilde{\mathbf{v}} ; \quad \tilde{\mathbf{v}}=\lambda \mathbf{v}+\mathbf{b}
$$

where $b$ is a translation vector, and $\lambda$ is a second-order tensor. It is known that affine transformations take points into points, lines into lines, and planes into planes. (Such transformations are also known as homogeneous deformations.) The tensor of transformation, $\lambda$, can always be expressed as the product of two second-order tensors: $\lambda=\mathbf{g} \cdot \mathbf{a}$, where $\mathbf{a}$ is a positive-definite, real, and symmetric, and $\mathbf{g}$ belongs to the three-dimensional group of real, orthogonal transformations, $O(3)$. In the following we shall be most interested in tensors of transformations in which a can be considered to differ negligibly from the second-order unit tensor; thus $\lambda \cong \mathbf{g}$. (This is equivalent to ignoring elastic strain in the lattice.)

The polycrystal is modeled as an aggreagate of crystallites filling a region of space, $R$. These crystallites have finite volume, and each material point belonging to any particular crystallite carries local structure associated with a specific affine transformation of the ideal reference crystal. The polycrystal will also contain material points for which this association is not possible, but we shall assume that this subregion has negligible volume.

Partition $O(3)$ into subsets $\delta \mathbf{g}_{i}$ such that

$$
\bigcup \delta g_{i}=O(3), \quad \delta g_{i} \cap \delta g_{j}=\emptyset \text { for } i \neq j \text {. }
$$

At point $\mathbf{x}$ in $R$ associates with $\delta g_{i}$ when there exists a neighborhood of $\mathbf{x}$, $N(\mathbf{x}) \subset R$, which carries structure related to the ideal reference crystal by an orthogonal transformation $\mathbf{g} \in \delta \mathbf{g}_{i}$, and a translation $\mathbf{b}$ which shall not be of further interest. Define the characteristic function $\chi$ associated with $\delta \mathbf{g}_{i}$ to be

$$
\chi_{\delta_{\mathbf{g}_{i}}}(\mathbf{x})= \begin{cases}1 & \text { if } \mathbf{x} \text { associates with } \delta \mathbf{g}_{i} \\ 0 & \text { otherwise }\end{cases}
$$

The volume fraction of crystallites associated with lattice orientation $\delta g_{i}$ in the polycrystal is just

$$
V\left(\delta \mathbf{g}_{i}\right) / V=\frac{1}{V} \int_{R} \chi_{\delta \mathbf{g}_{i}}(\mathbf{x}) d V
$$

Our primary interest lies not in representation of individual polycrystals, but rather in ensembles of polycrystals sharing a common thermo-mechanical processing path. We shall hereafter assume statistical homogeneity in the ensemble. For such an ensemble of polycrystals, each of which has volume $V$, the expected volume fraction of crystallites associated with lattice orientation $\delta \mathbf{g}_{i}$ is given by

$$
\begin{aligned}
\left\langle V\left(\delta \mathbf{g}_{i}\right)\right\rangle / V^{V} & =\left\langle\frac{1}{V} \int_{R} \chi_{\delta \mathbf{g}_{i}}(\mathbf{x}) d V\right\rangle \\
& =\frac{1}{V} \int_{R}\left\langle\chi_{\delta g_{i}}(\mathbf{x})\right\rangle d V \\
& =\left\langle\chi_{\delta g_{i}}(\mathbf{x})\right\rangle
\end{aligned}
$$

where $\left\langle\chi_{\delta_{\mathbf{g}_{i}}}(\mathbf{x})\right\rangle$ is independent of position $\mathbf{x}$. 


\subsection{Definition of the n-Point Probability Density Functions of Lattice Orientation}

The expectation value $\left\langle\chi_{\delta_{g_{i}}}(x)\right\rangle$ is closely related to the crystallite orientation distribution function $f: O(3) \rightarrow \mathbb{R}$ through the relation

$$
\left\langle V\left(\delta \mathbf{g}_{i}\right)\right\rangle / V=\left\langle\chi_{\delta_{\mathbf{g}_{i}}}(\mathbf{x})\right\rangle=\int_{\delta_{\mathbf{g}_{i}}} f(\mathbf{g}) d \mu
$$

where $d \mu$ is the invariant probability measure in $O(3)$. In the limit that our partitioning of $O(3)$ obtains infinitesimally small subsets $\mathrm{dg}$ of measure $\mu(\mathrm{dg})=$ $d \mu$, containing the orthogonal transformation $\mathrm{g}$, Eq. (6) can be expressed as

$$
\langle V(\mathbf{d g})\rangle / V=\left\langle\chi_{\mathrm{dg}}(\mathbf{x})\right\rangle=f(\mathbf{g}) d \mu .
$$

The orientation distribution function is normalized to unity:

$$
\int_{O(3)} f(\mathbf{g}) d \mu=1
$$

The preceding development can easily be extended to higher-order representations. For example, the expectation of $g_{0}$ and $g_{1}$ separated by $r_{1}$ in the ensemble of polycrystals is given by

$$
\left\langle\boldsymbol{\chi}_{\mathrm{dg}}(\mathbf{x}) \boldsymbol{\chi}_{\mathrm{dg}}\left(\mathbf{x}+\mathbf{r}_{1}\right)\right\rangle=f_{2}\left(\mathbf{g}_{0}, \mathbf{g}_{1} \mid \mathbf{r}_{1}\right) d \mu_{0} d \mu_{1},
$$

where $f_{2}$ is known as the two-point orientation coherence function (cf. Adams, et al. 1987; Adams, Wang and Morris, 1988; Adams and Onat, 1991). This can be generalized to consider the $(n+1)$-point probability density functions of lattice orientation since

$$
\left\langle\chi_{\mathrm{dg} g_{0}}(\mathbf{x}) \cdots \chi_{\mathrm{dg}_{n}}\left(\mathbf{x}+\mathbf{r}_{n}\right)\right\rangle=f_{n+1}\left(\mathbf{g}_{0}, \ldots, \mathbf{g}_{n} \mid r_{1}, \ldots, \mathbf{r}_{n}\right) d \mu_{0} \cdots d \mu_{0} .
$$

These functions have the property

$$
\int_{O(3)} f_{n+1}\left(\mathbf{g}_{0}, \ldots, \mathbf{g}_{n} \mid \mathbf{r}_{1}, \ldots, \mathbf{r}_{n}\right) d \mu_{n}=f_{n}\left(\mathbf{g}_{0}, \ldots, \mathbf{g}_{n-1} \mid \mathbf{r}_{1}, \ldots, \mathbf{r}_{n-1}\right) .
$$

Furthermore, they are normalized according to

$$
\int_{[O(3)]^{n+1}} f_{n+1}\left(\mathbf{g}_{0}, \ldots, \mathbf{g}_{n} \mid \mathbf{r}_{1}, \ldots, \mathbf{r}_{n}\right) d \mu_{0} \cdots d \mu_{n}=1,
$$

where the integration is over the product of $n+1$ copies of $O(3)$.

\subsection{Definition of the n-Point Correlation Tensors}

We are now in a position to define the $n$-point correlation tensors. A large class of physical and mechanical properties of crystals can be represented by tensors. Let $\mathbf{T}^{(k)}$ denote a tensor of order $k$ representing a material property of the ideal reference crystal. Relative to an orthonormal basis $\left\{\mathbf{e}_{i}\right\}$ the reference material tensor can be expressed in polyadic form as:

$$
\mathbf{T}^{\mathbf{0}(k)}=T_{j_{1} \cdots j_{k}}^{\mathbf{0}} \mathbf{e}_{j_{1}} \otimes \cdots \otimes \mathbf{e}_{j_{k}}
$$


where repeated indices imply summation according to the Einstein convention. In crystal, with its lattice transformed by $g$ relative to the ideal reference crystal, the same material property is represented by a tensor $\mathbf{T}^{(k)}=P_{\mathbf{g}} \mathbf{T}^{\mathbf{0}(k)}$,

$$
\begin{aligned}
\mathbf{T}^{(k)} & =T_{i_{1} \cdots i_{k}} \mathbf{e}_{i_{1}} \otimes \cdots \otimes \mathbf{e}_{i_{k}}=P_{\mathbf{g}} \mathbf{T}^{o(k)} \\
& =T_{j_{1} \cdots j_{k}}^{0} \mathbf{g e} \mathbf{e}_{j_{1}} \otimes \cdots \otimes \mathbf{g e}_{j_{k}} \\
& =g_{i_{1} j_{1}} \cdots g_{i_{k} j_{k}} T_{j_{1} \cdots j_{k}}^{\mathbf{0}} \mathbf{e}_{i_{1}} \otimes \cdots \otimes \mathbf{e}_{i_{k}},
\end{aligned}
$$

where we have set $g_{e_{j}}=g_{i j} \mathbf{e}_{i}$. Thus the coefficients of the property tensor in the transformed crystal are related to those of the ideal reference crystal by the relation

$$
T_{i_{1} \cdots i_{k}}=g_{i_{1} j_{1}} \cdots g_{i_{k} j_{k}} T_{j_{1} \cdots j_{k}}^{0}
$$

The average or mean tensor for the ensemble of polycrystals, $\left\langle\mathbf{T}^{(k)}\right\rangle$, is defined to be

$$
\left\langle\mathbf{T}^{(k)}\right\rangle=\int_{O(3)} f(\mathbf{g}) P_{\mathbf{g}} \mathbf{T}^{0(k)} d \mu .
$$

Because of assumed statistical homogeneity in the ensemble, the mean tensor is independent of position. The $n$-point correlation tensor is defined to be

$$
\begin{aligned}
& \left\langle\mathbf{T}(\mathbf{x}) \mathbf{T}\left(\mathbf{x}+\mathbf{r}_{1}\right) \cdots \mathbf{T}\left(\mathbf{x}+\mathbf{r}_{n-1}\right)\right\rangle \\
& \quad=\left\langle\mathbf{T}^{n}\right\rangle\left(\mathbf{r}_{1}, \ldots, \mathbf{r}_{n-1}\right) \\
& \quad=\int_{[O(3)]^{n}} f_{n}\left(\mathbf{g}_{0}, \mathbf{g}_{1}, \ldots, \mathbf{g}_{n-1} \mid \mathbf{r}_{1}, \ldots, \mathbf{r}_{n-1}\right) P_{\mathbf{g}_{0}} \mathbf{T}^{0} \cdots P_{\mathbf{g}_{n-1}} \mathbf{T}^{0} d \mu_{0} \cdots d \mu_{n-1},
\end{aligned}
$$

where we have now eliminated reference to the order of the material tensor for brevity.

The reader should note that the mean and correlation tensors can in principle be obtained directly from experimental measurements (viz, from microdiffraction methods; cf. Wright and Adams 1992; Kunze, Wright and Adams 1992). Consider an ensemble of $N$ polycrystals. Measure the tensor $\mathbf{T}$ at position $\mathbf{x}$ in each polycrystal. The mean tensor is then formed according to

$$
\langle\mathbf{T}(\mathbf{x})\rangle=\operatorname{Lim}_{N \rightarrow \infty} \sum_{n=1}^{N} \mathbf{T}(\mathbf{x})_{n} / N
$$

where $\mathbf{T}(\mathbf{x})_{n}$ denotes the measurement in the polycrystal denoted by $n$. The $n$-point correlation tensor is formed in analogous fashion by

$$
\left\langle\mathbf{T}^{n}\right\rangle\left(\mathbf{r}_{1}, \ldots, \mathbf{r}_{n-1}\right)=\operatorname{Lim}_{N \rightarrow \infty} \sum_{n=1}^{N} \mathbf{T}(\mathbf{x})_{n} \otimes \mathbf{T}\left(\mathbf{x}+\mathbf{r}_{1}\right)_{n} \otimes \cdots \otimes \mathbf{T}\left(\mathbf{x}+\mathbf{r}_{n-1}\right)_{n} / N
$$

In statistically homogeneous ensembles the mean and correlation tensors are independent of position $\mathbf{x}$. It should be evident that correlations of all types of mixed tensors, such as $\langle$ T S T $\rangle\left(\mathbf{r}_{1}, \mathbf{r}_{2}\right)$, are defined in the same manner.

It is important to note that it is not generally possible to construct mean and correlation tensors for a second material tensor (e.g. of differing rank) from those of a first material tensor. For this reason the $n$-point probability density functions $f_{n}$ are considered to be more fundamental representations of microstructure than 
the correlation tensors themselves. However, in specific problems it may be easiest to deal with the particular $n$-point correlation tensors directly.

\subsection{Model Microstructures Exhibiting Only Crystallite-Scale Auto-Coherence}

It is possible that in some microstructures the geometrical features of the constituent crystallities may be statistically uncorrelated with lattice orientation. In other words, grain size and shape, and other geometrical features of the microstructure may be statistically decoupled from lattice orientation. It is also possible that some microstructures may exhibit no measureable coherence between neighboring crystallites. An example of such microstructure exhibiting these two features was recently described by Wang, Morris and Adams (1990). When such microstructure is present a major simplification occurs in the structure of the probability density functions of lattice orientation.

The two-point correlation function must have the form

$$
f_{2}\left(g_{0}, g_{1} \mid \mathbf{r}_{1}\right)=\phi\left(\mathbf{r}_{1}\right) f\left(g_{0}\right) \delta\left(g_{0}, g_{1}\right)+\left(1-\phi\left(\mathbf{r}_{1}\right)\right) f\left(g_{0}\right) f\left(g_{1}\right),
$$

where $\delta\left(g_{0}, g_{1}\right)$ is the Dirac function centered about $g_{0}$, and $\phi\left(\mathbf{r}_{1}\right)$ is the probability that the random point $x$ and $x+r_{1}$ hit the same grain. In the model microstructure described, $\phi\left(\mathbf{r}_{1}\right)$ is independent of $g_{0}$ and $g_{1}$. We note that this form of the two-point function satisfies the normalization conditions expressed in (12).

Now consider the form of the three-point correlation function. Let $\Theta\left(\mathbf{r}_{1}, \mathbf{r}_{2}\right)$ be the probability that the random point $x$, and points $x+r_{1}$ and $x+r_{2}$ all hit different crystallites (i.e., different lattice orientations). Let $\vartheta(m, n)$ be the probability that $\mathbf{x}+\mathbf{m}$ and $\mathbf{x}+\mathbf{n}$ fall in the same crystallite while the random point $\mathbf{x}$ falls in a separate crystallite. With these definitions the three-point correlation function has the form

$$
\begin{aligned}
& f_{3}\left(g_{0}, g_{1}, g_{2} \mid \mathbf{r}_{1}, \mathbf{r}_{2}\right) \\
&= \Theta\left(\mathbf{r}_{1}, \mathbf{r}_{2}\right) f\left(g_{0}\right) f\left(g_{1}\right) f\left(g_{2}\right)+\vartheta\left(-\mathbf{r}_{2}, \mathbf{r}_{1}-\mathbf{r}_{2}\right) f\left(g_{0}\right) \delta\left(g_{0}, g_{1}\right) f\left(g_{2}\right) \\
&+\vartheta\left(\mathbf{r}_{1}, \mathbf{r}_{2}\right) f\left(g_{0}\right) f\left(g_{1}\right) \delta\left(g_{1}, g_{2}\right)+\vartheta\left(-\mathbf{r}_{1}, \mathbf{r}_{2}-\mathbf{r}_{1}\right) f\left(g_{2}\right) \delta\left(g_{2}, g_{0}\right) f\left(g_{1}\right) \\
&+\left(1-\Theta\left(\mathbf{r}_{1}, \mathbf{r}_{2}\right)-\vartheta\left(-\mathbf{r}_{2}, \mathbf{r}_{1}-\mathbf{r}_{2}\right)-\vartheta\left(\mathbf{r}_{1}, \mathbf{r}_{2}\right)-\vartheta\left(-\mathbf{r}_{1}, \mathbf{r}_{2}-\mathbf{r}_{1}\right)\right) f\left(g_{0}\right) \delta\left(g_{0}, g_{1}, g_{2}\right),
\end{aligned}
$$

where $\delta\left(g_{0}, g_{1}, g_{2}\right)$ is a Dirac distribution on $O(3) \times O(3) \times O(3)$ defined by

$$
\int_{\Omega_{1}} \int_{\Omega_{2}} \delta\left(g_{0}, g_{1}, g_{2}\right) d \mu_{1} d \mu_{2}=1 \quad \text { when } g_{0} \in \Omega_{1}, \Omega_{2} ; 0 \text { otherwise. }
$$

In this expression $\Omega_{1}, \Omega_{2} \subset O(3)$.

\section{REPRESENTATIONS OF GROUPS}

In this section we recall some aspects of the theory of group representations which are required in the subsequent development. We focus on finite and compact groups. 
Let $G$ be a group. We shall say that we have an $n$-dimensional matrix representation $g \rightarrow M(g)$ of $G$ if to each element $g \in G$ there associates an invertible linear $n \times n$ matrix $M(g)$ such that the product of elements of the group corresponds to the product of their matrices. That is to say, $M\left(g_{1}\right) M\left(g_{2}\right)=$ $M\left(g_{1} g_{2}\right)$. It is also required, for the identity element of the group $g=i d$, that there corresponds the identity transformation $M(i d)=I_{n}$, which is the $n \times n$ diagonal matrix consisting of 1's down the diagonal and 0's elsewhere. It follows that $M\left(g^{-1}\right)=(M(g))^{-1}$ for all $g \in G$.

If $g \rightarrow M(g)$ is a matrix representation of $G$, then for any invertible $(n \times n)$ matrix $A$ we can construct another representation $\hat{M}(g)=A^{-1} M(g) A$. Since $M$ and $\hat{M}$ can be obtained from one-another by simple conjugation, their properties are the same, and they are called equivalent (isomorphic). In representation theory, equivalent representations are considered to be the same representation.

Now consider a linear action of the group $G$ in a vector space $V$ of dimension $n$. Thus, to every element $g \in G$ is associated a non-singular linear transformation $T_{g}: V \rightarrow V$ with properties $T_{g 1} T_{g 2}=T_{g 1 g 2}$ and $T_{\text {id }}=\mathrm{id}_{V}$ where id $V$ is the identity element of $V$. Taking any basis $B$ of $V$, we can express the operator $T_{g}$ by its matrix $\left[T_{g}\right]^{B}=M_{T}^{B}(g)$ in this basis; note that thereby we define an $n$-dimensional matrix representation of $G$. Representations obtained by means of different bases are equivalent since for any pair of bases $B 1$ and $B 2$ there is defined a transition matrix $S_{B 1 B 2}$ such that $M_{T}^{B 2}(g)=S_{B 1 B 2}^{-1} M_{T}^{B 1}(g) S_{B 1 B 2}$.

Two operations are defined for matrix representations: the direct sum, and the tensor product. Given two representations $M_{1}$ and $M_{2}$ of dimensions $n_{1}$ and $n_{2}$, respectively, their direct sum, $M=M_{1} \oplus M_{2}$, is constructed according to

$$
M(g)=\left[\begin{array}{cc}
M_{1}(g) & 0 \\
0 & M_{2}(g)
\end{array}\right]
$$

The new representation $M$ is of dimension $n_{1}+n_{2}$. Notice that, strictly speaking $M_{1} \oplus M_{2} \neq M_{2} \oplus M_{1}$; however, these two representations are obviously isomorphic, and therefore indistinguishable in representation theory.

The tensor product of two matrix representations obtains from the Kronecker cross-product of two matrices:

$$
\begin{aligned}
\tilde{M}(g) & =M_{1}(g) \otimes M_{2}(g)= \\
& =\left[\begin{array}{cccc}
m_{1}^{(11)}(g) M_{2}(g) & m_{1}^{(12)}(g) M_{2}(g) & \cdots & m_{1}^{\left(1 n_{1}\right)}(g) M_{2}(g) \\
m_{1}^{(21)}(g) M_{2}(g) & m_{1}^{(22)}(g) M_{2}(g) & \cdots & m_{1}^{\left(2 n_{1}\right)}(g) M_{2}(g) \\
\vdots & \vdots & \vdots & \vdots \\
m_{1}^{\left(n_{1} 1\right)}(g) M_{2}(g) & m_{1}^{\left(n_{1}\right)}(g) M_{2}(g) & \cdots & m_{1}^{\left(n_{1} n_{1}\right)}(g) M_{2}(g)
\end{array}\right]
\end{aligned}
$$

where $m_{1}^{(i j)}(g)$ denote the $i j$ entry of $M_{1}(g)$. As in the case of the direct sum, the tensor product is not commutative: $M_{1} \otimes M_{2} \neq M_{2} \otimes M_{1}$. In the context of representation theory, however, they are isomorphic.

Let $V, W$ be vector spaces. Let $v_{1}, \ldots, v_{n}$ be a basis of $V$ and $w_{1}, \ldots, w_{m}$ be a basis of $W$. The tensor product $V \otimes W$ is defined as the space whose basis is $v_{i} \otimes w_{j}, 1 \leq i \leq n, 1 \leq j \leq m$. For two vectors

$$
v=\sum_{i} a_{i} v_{i} \in V \quad w=\sum_{j} b_{j} w_{j} \in W
$$


we can define the cross product $v \otimes w \in V \otimes W$ by

$$
v \otimes w=\sum_{i, j} a_{i} b_{j} v_{i} \otimes w_{j}
$$

If there is a linear action of a group $G$ in $V$ and in $W$, we can naturally define an action of $G$ in $V \otimes W$ by $g\left(v_{i} \otimes w_{j}\right)=\left(g v_{i}\right) \otimes\left(g w_{j}\right), g \in G, 1 \leq i \leq n, 1 \leq j \leq m$. Note that this action is independent of the choice of basis in $V$ and $W$, although a choice has been used in the definition. It can also be seen that the matrix representation of this action is equal to the tensor product of matrix representations of the actions of $G$ in $V$ and $W: M=M_{1} \otimes M_{2}$.

The foregoing construction enables a definition of the power $V^{\otimes M}$ of a vector space $V$, and an action of $G$ in $V^{\otimes M}$, assuming one exists in $V$. A basis of $V^{\otimes M}$ is $\left\{v_{j_{1}} \otimes \cdots \otimes v_{j_{M}}\right\}$ where $\left\{v_{j}\right\}$ is a basis of $V$. Define the $M$ th symmetric power of $V$ as a subspace $S^{M} V \subset V^{\otimes M}$ which consists of all possible linear combinations

$$
\sum_{j_{1} \cdots j_{M}} a_{j_{1} \cdots j_{M}} v_{j_{1}} \otimes \cdots \otimes v_{j_{M}}
$$

where $a_{j_{1} \cdots j_{M}}$ is symmetric with respect to permutations of its indices. Obviously, $S^{M} V$ is an invariant subspace under any linear action of a group $G$ in $V^{\otimes M}$ arising from an action of $G$ in $V$. It follows that if we have a linear action of $G$ in $V$, we also have one in $S^{M} V$. The dimension of $S^{M} V$ is $\left(\begin{array}{c}M+n-1 \\ n-1\end{array}\right)$ where $n$ is the
dimension of $V$.

A representation $M$ is said to be reducible if it is possible to find an equivalent representation $\hat{M}$ such that $\hat{M}(g)$ for all $g$ belonging to the group $G$ has the form

$$
\hat{M}(g)=\left[\begin{array}{cc}
M_{1}(g) & Y(g) \\
0 & M_{2}(g)
\end{array}\right]
$$

where $M_{1}(g)$ and $M_{2}(g)$ are $\left(n_{1} \times n_{1}\right)$ and $\left(n_{2} \times n_{2}\right)$ matrix representations, respectively. $M_{1}(g)$ is called a subrepresentation of $M(g)$, and $M_{2}(g)$ is called the factor representation of $M$ by $M_{1}$. If it is not possible to find an equivalent representation of this form, the representation is called irreducible. For the groups of interest in this paper (i.e., finite and compact groups) any representation is isomorphic to a direct sum of irredubile representations. This decomposition is unique up to the order of the summands. In other words, Eq. (28) can always be reduced to the form of Eq. (23) with $Y(g)=0$, where $M_{1}(g)$ and $M_{2}(g)$ are new representations of $G$.

We conclude this general discussion by mentioning invariants. Suppose we have a linear action $g \rightarrow T_{g}$ of a group $G$ in a vector space $V$. Let $\Sigma$ be another group (this may be $G$ itself or any subgroup of $G$, or a group of different nature) that also acts linearly on $V: \sigma \rightarrow S_{\sigma}: V \rightarrow V$. Then we can form a particular subspace of $V, V^{\Sigma}=\{v \in V \mid$ for all $\sigma \in \Sigma, \sigma \cdot v=v\}$. It is called the subspace of $\Sigma$-invariants in $V$. Assume that $S$ commutes with $T$; that is, $S_{\sigma} T_{g}=T_{g} S_{\sigma}$ for all $\sigma \in \Sigma$ and $g \in G$. Then the subspace $V^{\Sigma}$ will be invariant under the action of $G$. It gives rise to a subrepresentation $M^{\Sigma}$ of the matrix representation $M$ of the action of $G$ in $V$. 


\subsection{Representations of $O(3)$}

One of the central problems of representation theory is to classify the irreducible representations of a given group. For finite and compact groups this problem is generally solved. Here we shall state a few results of this classification for the orthogonal group in $\mathbb{R}^{3}, O(3)$.

It is known (cf. Broecker and tom Dieck 1985) that for any integer $m \geq 0$ there exists exactly two non-isomorphic irreducible representations of $O(3)$ having dimension $k=2 m+1$. Even dimensional irreducible representations do not exist. Let us denote these two representations by $M_{k}^{+}$and $M_{k}^{-}$where $k$ is now any positive odd integer. Also, let inv denote the inversion element of the orthogonal group:

$$
\operatorname{inv}=\left[\begin{array}{rrr}
-1 & 0 & 0 \\
0 & -1 & 0 \\
0 & 0 & -1
\end{array}\right] \in O(3)
$$

In the representation $M_{k}^{+}$the group element inv maps to $I_{k}$, the $k$-dimensional identity matrix. For this reason we can think of $M_{k}^{+}$as being the $k$-dimensional representation of the quotient group $O(3) / \Pi \cong S O(3)$ where $\Pi$ is the group of two elements $\{i d, i n v\}$. $(O(3) / \Pi$ is the manifold (collection) of all physically distinctive (right or left) cosets of $\Pi$ in $O(3)$, and $S O(3)$ is the three-dimensional special orthogonal group of transformations which have determinant equal to +1.) The representations $M_{k}^{-}$are obtained from $M_{k}^{+}$, for all $g \in G$, by the relation $M_{k}^{-}(g)=M_{k}^{+}(g) \cdot \operatorname{det}(g)$.

An important example of a reducible matrix representation of $O(3)$ is the representation in tensors of rank $l$ on $\mathbb{R}^{3}$. This representation associates to an element $g \in O(3)$ the matrix connecting the coefficients of any tensor $T^{0}$ of rank $l$ and the transformed tensor $P_{g} T^{0}$ (cf. Eq. (15)). This representation has dimension $3^{l}$, and it is isomorphic to $M_{3}^{-} \oplus \cdots l$-times $\cdots \oplus M_{3}^{-}\left(M_{3}^{-}\right)^{\otimes l}$.

Irreducible representations of $O(3)$ can be realized by considering completely symmetric and traceless tensors. The coefficients of such tensors are completely symmetric according to

$$
T_{j_{1} j_{2} \cdots j_{l}}=T_{p\left[j_{1} j_{2} \cdots j_{i}\right]}
$$

where $p[()]$ denotes permutation of ( ). Furthermore they are traceless according to

$$
T_{j_{n} j_{n} \cdots j_{l}}=0 \text {. }
$$

This leaves $2 l+1$ independent components.

Now consider transformations of the form $\mathbf{T}=P_{g} \mathbf{T}^{0}, g \in O(3)$, as described in Eq. (15). If $\mathbf{T}^{0}$ is completely symmetric and traceless, then it is easily demonstrated that $\mathbf{T}$ will also be completely symmetric and traceless. The matrix of coefficients connecting the independent components of $\mathbf{T}^{0}$ with those of the transformed tensor $\mathbf{T}$ satisfies all of the required properties to qualify as a $2 l+1$ dimensional irreducible representation of $O(3)$. It is isomorphic to $M_{2 l+1}^{+}$if $l$ is even, and to $M_{2 l+1}^{-}$if $l$ is odd.

One central problem of representation theory is the decomposition of the tensor product. If $M_{1}(g)$ and $M_{2}(g)$ are irreducible representations of a group $G$, the representation $M_{1}(g) \otimes M_{2}(g)$ is usually not irreducible. It follows that it can 
be decomposed into a direct sum of irreducible representations. Any particular irreducible repesentation may occur several times in this direct sum. The number of times it occurs is called its multiplicity. The multiplicities of occurrence of irreducible representations in tensor products are called Clebsch-Gordan coefficients, and the corresponding decomposition is called the Clebsch-Gordan decomposition.

The Clebsch-Gordan decomposition for $O(3)$ is well known. Given two representations $M_{k}^{ \pm}$and $M_{p}^{ \pm}$, the decomposition is

$$
\begin{aligned}
M_{k}^{ \pm} \otimes M_{p}^{ \pm} & \cong \bigoplus_{j=1}^{\min (k, p)} M_{k+p+1-2 j}^{ \pm} \\
& =M_{k+p-1}^{ \pm} \oplus M_{k+p-3}^{ \pm} \oplus \cdots \oplus M_{|k-p|+1}^{ \pm},
\end{aligned}
$$

where the combination of,++ or,-- on the left-hand side of the equation results in + in all terms on the right-hand side, otherwise - appears in all terms on the right-hand side. The multiplicities are either 0 or 1 when considering $O(3)$.

\subsection{Representations In the Space of Square-Integrable Functions, $L^{2}(X)$}

There is a class of infinite-dimensional representations that will be of interest. Of interest is the action of $O(3)$ in spaces of square-integrable functions, $L^{2}(X)$, defined over the domain $X=O(3) / \Gamma$ where $\Gamma$ is a closed subgroup of $O(3)$. The action of $O(3)$ in $L^{2}(X)$ is defined by

$$
P_{g} f(x)=f\left(g^{-1} x\right), \quad g \in O(3), \quad x \in X .
$$

The relationship between $f(x)$ and $P_{g} f(x)$ is an infinite dimensional matrix $M_{\infty}$ in any given basis of $L^{2}(X)$. It gives rise to an infinite dimensional matrix representation of $O(3)$. A well known result of representation theory insures that by appropriate change of the basis it is possible to reduce this matrix to a block-diagonal matrix consisting of blocks of finite size. Stated another way, the action of $O(3)$ on $L^{2}(X)$ reduces to an infinite sum of finite, odd-dimensional, irreducible representations. Thus,

$$
M_{\infty} \cong \bigoplus_{k=0}^{\infty} m_{k}^{ \pm} M_{2 k+1}^{ \pm}
$$

where $m_{k}^{ \pm}$is the multiplicity of occurrence of the representation $M_{2 k+1}^{ \pm}$in $M_{\infty}$.

\subsection{Tensorial Representations of $f: X \rightarrow \mathbb{R}$}

The decomposition of $L^{2}(X)$ into irreducible representations enables a Fourier analysis on the space $X=O(3) / \Gamma$. It is known (see Adams, Guidi, Boehler and Onat 1992, and Guidi, Adams and Onat 1992) that any function $f: X \rightarrow \mathbb{R}$ can be expressed as

$$
f(x)=\sum_{k=1}^{\infty}\left(\sum_{i=1}^{m_{k}^{+}} \sum_{j=-k}^{k} C_{j}^{i, k,+}(f) \varphi_{j}^{i, k,+}(x)+\sum_{i=1}^{m_{k}^{-}} \sum_{j=-k}^{k} C_{j}^{i, k,-}(f) \varphi_{j}^{i, k,-}(x)\right)
$$

where the vector $\varphi_{j}^{i, k, \pm}$ transforms according to the $M_{2 k+1}^{ \pm}$component of $M_{\infty}$ 
under the action of $O(3)$. This decomposition may not be unique since the multiplicities $\boldsymbol{m}_{k}^{ \pm}$will generally exceed 1 , but a decomposition always exists.

$\varphi^{i, k, \pm}=\left\{\varphi_{-k}^{i, k, \pm}, \ldots, \varphi_{+k}^{i, k, \pm}\right\}$ can be interpreted as vector functions on $X$ with values in the space of completely symmetric and traceless tensors of rank $k$ on $\mathbb{R}^{3}$. They are easily constructed from the tensor transformation expressed in Eq. (15). It is also readily shown that the coefficients $C_{j}^{i, k, \pm}(f)$ are also associated with symmetric and traceless tensors $C^{i, k, \pm}(f)$ of order $k$. Then relation (35) can be rewritten as

$$
f(x)=\sum_{k=0}^{\infty}\left(\sum_{i=1}^{m_{k}^{+}} C^{i, k,+}(f) \cdot \varphi^{i, k,+}(x)+\sum_{i=1}^{m_{k}} C^{i, k,-}(f) \cdot \varphi^{i, k,-}(x)\right)
$$

where the product $C \cdot \varphi$ is understood to be the contraction of tensors.

With regard to the choice of decomposition, we impose orthogonality and normalization conditions on the invariant integration of products of functions:

$$
\begin{gathered}
\int_{X} \varphi_{p_{1} \cdots p_{k}}^{i, k, \pm}(x) \otimes \varphi_{p_{1} \cdots p_{k}}^{i, k, \pm}(x) d \mu=1, \\
\int_{X} \varphi^{i, k, \pm}(x) \otimes \varphi^{j, k, \pm}(x) d \mu=0 \quad \text { unless } i=j \\
\int_{X} \varphi^{i, k,+}(x) \otimes \varphi^{j, k,-}(x) d \mu=0 .
\end{gathered}
$$

Any set of tensor valued functions satisfying these conditions constitutes a coordinate-free, tensorial, orthogonal representation of $L^{2}(X)$. (If multiplicities $>1$ are involved, it is still not unique, however.)

\section{REPRESENTATIONS OF CORRELATION TENSORS}

We now formulate the central problem of the paper. Let $\mathbf{T}$ be a tensorial observable of rank $k$. Form the correlation functions $\left\langle\mathbf{T}^{M}\right\rangle\left(\mathbf{r}_{1}, \ldots, \mathbf{r}_{M-1}\right)$, which are tensors of rank $M k$. Let $\Sigma$ denote the indicial symmetry characteristics of $\mathbf{T}$ $\left(\Sigma \subset S_{k}\right.$, the symmetric group of degree $\left.k\right)$. By $\Gamma$ we denote the symmetry subgroup of the crystal lattice $(\Gamma \subset O(3))$, and by $P$ we indicate the symmetry subgroup associated with thermomechanical processing $(P \subset O(3))$. The physical meaning of $P$ is that processing often induces statistical symmetries in the microstructure reflecting symmetries in the mechanical, thermal and chemical forces employed in processing. The question of focal interest here is to determine the number of independent parameters that fix the value of $\left\langle\mathbf{T}^{M}\right\rangle\left(\mathbf{r}_{1}, \ldots, \mathbf{r}_{M-1}\right)$ for specified $\mathbf{r}_{1}, \ldots, \mathbf{r}_{M-1} \in \mathbb{R}^{3}$. In other words, we seek to find the appropriate "residence space" for such correlation tensors.

We already know that the matrix representation of the action of $O(3)$ on the space $V(k)$ of tensors of rank $k$ is isomorphic to $M_{3}^{-\otimes k}$. The tensor $\mathbf{T}$ that is of interest to us is invariant with respect to the action of $\Sigma$, and it resides in the space

$$
\mathscr{T}(k, \Sigma)=_{\text {def }} V(k)^{\Sigma}=\left\{v \in V(k) \mid \text { for all } \sigma \in \Sigma, S_{\sigma} v=v\right\}
$$


Since the actions of $O(3)$ and $\Sigma$ in $V(k)$ commute, $V(k)^{\Sigma}$ inherits a linear $O(3)$-action from $V(k)$, and the matrix representation of this action is $\left(M_{3}^{-\otimes k}\right)^{\Sigma}$. The structure of this representation can be easily determined for all cases of interest. Its decomposition into a direct sum of irreducible representations has the form

$$
\left(M_{3}^{-\otimes k}\right)=\bigoplus_{j=0}^{k} C_{j}(k, \Sigma) M_{2 j+1}^{ \pm}
$$

where the sign of $M$, on the right hand side, is positive when $k$ is even, otherwise it is negative. The coefficients $C_{j}(k, \Sigma)$ are positive integers, or zero.

We consider material tensors $\mathbf{T}$ which are symmetric with respect to a subgroup $\Gamma$ characteristic of the crystal lattice. It follows that $T$ lies in the space of $\Gamma$-invariants of $\mathscr{T}(k, \Sigma)$; or in other words, in the space $\mathscr{T}(k, \Sigma)^{\Gamma} . \mathscr{T}(k, \Sigma)^{\Gamma}$ is not, in general, an $O(3)$-invariant subspace since the actions of $\Gamma$ and $O(3)$ in $\mathscr{T}(k, \Sigma)$ need not commute. We shall therefore restrict our attention to the $O(3)$-invariant subspace $W(k, \Sigma, \Gamma)$ of $\mathscr{T}(k, \Sigma)$ that is generated by the subspace $\mathscr{T}(k, \Sigma)^{\Gamma}$. In other words, $W(k, \Sigma, \Gamma)$ is defined to be the smallest invariant subspace of $O(3)$ in $\mathscr{T}(k, \Sigma)$ which contains $\mathscr{T}(k, \Sigma)^{\Gamma}$.

There is an easy rule which allows us to construct $W(k, \Sigma, \Gamma)$ from $\mathscr{T}(k, \Sigma)$. Let us say that $M_{2 k+1}^{ \pm}$is "empty" with respect to $\Gamma$ if the matrices $M_{2 k+1}^{ \pm}(\gamma)$, $\gamma \in \Gamma$, do not have a non-zero common invariant vector. A criterion for $M_{2 k+1}^{ \pm}$to be empty is that $m_{k}^{ \pm}=0$, where $m_{k}^{ \pm}$is the multiplicity of occurrence of $M_{2 k+1}^{ \pm}$in $L^{2}(X)$, and $X=O(3) / \Gamma$. The procedure for constructing $W(k, \Sigma, \Gamma)$ is as follows: (1) Decompose $\mathscr{T}(k, \Sigma)$ into the direct sum of irreducible representations of $O(3)$ using the Clebsch-Gordan rule, then (2) Eliminate any terms which are empty with respect to $\Gamma$. The result is exactly $W(k, \Sigma, \Gamma)$. The matrix representation of the action of $O(3)$ in the subspace, denoted by $M W(k, \Sigma, \Gamma)$ decomposes according to

$$
\mu W(k, \Sigma, \Gamma)=\bigoplus_{j=0}^{k} C_{j}(k, \Sigma, \Gamma) M_{2 j+1}^{ \pm}
$$

As we extend these ideas to the correlation tensor $\left\langle\mathbf{T}^{M}\right\rangle\left(\mathbf{r}_{1}, \ldots, \mathbf{r}_{M-1}\right)$, it is natural to regard it as an element of the space $W(k, \Sigma, \Gamma)^{\otimes M}$. This is the residence space for correlation tensors $\left\langle\mathbf{T}^{M}\right\rangle$ when $M>2$.

When $M=1$ we must take into account the processing symmetry, and the residence space of $\langle T\rangle$ is $W(k, \Sigma, \Gamma)^{P}$. When $M=2$, if the processing symmetry group $P$ contains the element inv, then the space where $\langle\mathbf{T T}\rangle(\mathbf{r})$ lies will be $S^{2} W(k, \Sigma, \Gamma)$, and it follows that $\operatorname{dim}\left[S^{2} W(k, \Sigma, \Gamma)\right]=\operatorname{dim}[W(k, \Sigma, \Gamma)] \times$ $(\operatorname{dim}[W(k, \Sigma, \Gamma)]+1) / 2$.

For the model microstructure described in Section 2.3 the number of independent parameters can be further reduced. As before the tensor $\left\langle\mathbf{T}^{M}\right\rangle$ lies in the space $\mathscr{T}(k, \Sigma)^{\otimes M}$, and its independent parameters can be found as described above. However, if $M \geq 2$, some of these parameters occur in $\left\langle\mathbf{T}^{N}\right\rangle$, for $N<M$. More precisely, use the notation $\left\langle\mathrm{T}^{M}\right\rangle^{0}$ to represent the mean value of the $M$ th tensor product of $\mathbf{T}$ :

$$
\left\langle\mathbf{T}^{M}\right\rangle^{\mathbf{0}}=\langle\mathbf{T}(\mathbf{x}) \otimes \cdots M \text { times } \cdots \otimes \mathbf{T}(\mathbf{x})\rangle .
$$

It is evident from Eqs. (17), (20) and (21) that for the model microstructure

$$
\left\langle\mathbf{T}^{2}\right\rangle\left(\mathbf{r}_{1}\right)=\phi\left(\mathbf{r}_{1}\right)\left\langle\mathbf{T}^{2}\right\rangle^{0}+\left(1-\phi\left(\mathbf{r}_{1}\right)\right)\langle\mathbf{T}\rangle^{2},
$$


and

$$
\begin{aligned}
\left\langle\mathbf{T}^{3}\right\rangle\left(\mathbf{r}_{1}, \mathbf{r}_{2}\right)= & \Theta\left(\mathbf{r}_{1}, \mathbf{r}_{2}\right)\langle\mathbf{T}\rangle^{3}+\vartheta\left(-\mathbf{r}_{2}, \mathbf{r}_{1}-\mathbf{r}_{2}\right)\left\langle\mathbf{T}^{2}\right\rangle^{0} \otimes\langle\mathbf{T}\rangle \\
& +\vartheta\left(\mathbf{r}_{1}, \mathbf{r}_{2}\right)\langle\mathbf{T}\rangle \otimes\left\langle\mathbf{T}^{2}\right\rangle^{0}+\vartheta\left(-\mathbf{r}_{1}, \mathbf{r}_{2}-\mathbf{r}_{1}\right)\langle\mathbf{T} \otimes\langle\mathbf{T}\rangle \otimes \mathbf{T}\rangle^{\mathbf{0}} \\
& +\left(1-\Theta\left(\mathbf{r}_{1}, \mathbf{r}_{2}\right)-\vartheta\left(-\mathbf{r}_{2}, \mathbf{r}_{1}-\mathbf{r}_{2}\right)-\vartheta\left(\mathbf{r}_{1}, \mathbf{r}_{2}\right)-\vartheta\left(-\mathbf{r}_{1}, \mathbf{r}_{2}, \mathbf{r}_{1}\right)\right)\left\langle\mathbf{T}^{3}\right\rangle^{0}
\end{aligned}
$$

Note that $\langle\mathbf{T} \otimes\langle\mathbf{T}\rangle \otimes \mathbf{T}\rangle_{I J L}^{0}=\left\langle\mathbf{T}^{2}\right\rangle^{0} \otimes\langle\mathbf{T}\rangle_{I L J}$ where $I=i_{1} \cdots i_{k}, J=j_{1} \cdots j_{k}$, and $L=l_{1} \cdots l_{k}$.

Thus, the only additional information required by the two-point correlation tensor, beyond the simple mean value of the tensor itself, is the mean value of the tensor squared (i.e. $\left.\left\langle\mathbf{T}^{2}\right\rangle^{0}\right)$. For the three-point correlation tensor the only additional information required, beyond what is required for the two-point correlation tensor, is the mean value of the tensor cubed (i.e. $\left.\left\langle\mathbf{T}^{3}\right\rangle^{0}\right)$. This trend obviously repeats itself for higher-order correlation tensors. The only new information required by the $M$ th correlation tensor, beyond the $(M-1)$ th and lower-order correlation tensors, is $\left\langle\mathbf{T}^{M}\right\rangle^{0}$.

Mean values of the form $\left\langle\mathbf{T}^{M}\right\rangle^{0}$ occurring in the model microstructure are defined by many fewer independent parameters than $\left\langle\mathbf{T}^{M}\right\rangle$. In particular, there is no dependence upon $\mathbf{r}_{1}, \ldots, \mathbf{r}_{M-1}$. It is also clear that $\left\langle\mathbf{T}^{M}\right\rangle^{0}$ is symmetric with respect to permutations of its $M$ factors. Moreover, since $\mathbf{T}$ lies in $\mathscr{T}(k, \Sigma)^{\boldsymbol{\Gamma}}, \mathbf{T}^{M}$ lies in $S^{M}\left(\mathscr{T}(k, \Sigma)^{\Gamma}\right)$, so $\left\langle\mathbf{T}^{M}\right\rangle^{0}$ must lie in the minimal $O(3)$-subrepresentation of $S^{M} W(k, \Sigma, \Gamma)$ that contains $S^{M}\left(\mathscr{T}(k, \Sigma)^{\Gamma}\right)$. This minimal subrepresentation we denote by $U(k, \Sigma, \Gamma, M)$. As before, in order to find $U$, we can decompose $S^{M} W(k, \Sigma, \Gamma)$ into a direct sum of irreducible subrepresentations, and then eliminate those which are empty with respect to $\Gamma$. In general, this will still leave us with a larger subrepresentation than $U$; there may be some non-empty components of $S^{M} W$ that do not occur in $U$. Indeed, for instance if $\mathscr{T}(k, \Sigma)^{r}$ is 1-dimensional, every irreducible $O(3)$-representation (even non-empty) can occur in the decomposition of $U$ no more than once. This illustrates that finding the space $U$ for nontrivial $\Gamma$ may require a case-by-case consideration, and cannot be reduced to a general rule.

The processing symmetry reduces the space $U(k, \Sigma, \Gamma, M)$ to the space of invariants $U(k, \Sigma, \Gamma, M)^{P}$, which is exactly the space of independent parameters of $\left\langle\mathbf{T}^{M}\right\rangle^{0}$. This answers the fundamental question addressed by this paper.

\subsection{Examples from Representations of Material Tensors of Order Two}

4.1.1. Consider symmetric material tensors $\mathrm{T}$ of order two when no lattice symmetry is present. Thus, $k=2, \Sigma=S_{2}$ (the group of permutations of two objects), and $\Gamma$ is the trivial group $I$ consisting of the identity element. The space $W\left(2, S_{2}, I\right)$ is just the space of symmetric tensors of rank two. The representation of $O(3)$ in this space is isomorphic to $M_{1}^{+} \oplus M_{5}^{+}$and its dimension is 6 . (The reader may find it convenient to think of the stress or strain tensors which are symmetric, and can be expressed in terms of a five-dimensional deviatoric tensor and a one-dimensional trace.)

4.1.1.a. When the processing symmetry group is trivial, then the residence space of $\left\langle\mathbf{T}^{M}\right\rangle$ is the $6^{M}$ dimensional space $W\left(2, S_{2}, I\right)^{\otimes M}$ whose independent para- 
meters are the entries $\left\langle\mathbf{T}^{M}\right\rangle_{i_{1} j_{1} i_{2} j_{2} \cdots i_{M} j_{M}}$ where $i_{r} \leq j_{r}$. The remaining entries of the correlation tensor are obtained by symmetry.

4.1.1.b. When the processing symmetry group is the orthotropic group,

$$
P=D_{2 h}=\left\{\left[\begin{array}{rrr} 
\pm 1 & 0 & 0 \\
0 & \pm 1 & 0 \\
0 & 0 & \pm 1
\end{array}\right]\right\}
$$

then for $M>2$ the answers are precisely the same as when $P$ is equal to the trivial group $I$. However, when $M=1$ the residence space of $\langle T\rangle$ is $W\left(2, S_{2}, I\right)^{D_{2 h}}$. This space is three-dimensional, and the corresponding $D_{2 h}$-invariant tensors are all diagonal tensors. The independent parameters are $\langle\mathbf{T}\rangle_{11},\langle\mathbf{T}\rangle_{22}$, and $\langle\mathbf{T}\rangle_{33}$ with all other entries being zero. When $M=2$ the residence space of $\left\langle\mathbf{T}^{2}\right\rangle$ is $S^{2} W\left(2, S_{2}, I\right)$, recalling that

$$
S^{2} V \subset V \otimes V, \quad S^{2} V=\left\{\sum a_{i j} v_{i} \otimes v_{j} \mid v_{i}, v_{j} \in V, a_{i j}=a_{j i}\right\} .
$$

It is evident that $S^{2} W\left(2, S_{2}, I\right)$ is 21 -dimensional. In this case the independent parameters are $\left\langle\mathbf{T}^{2}\right\rangle_{i j k l}$, with $i \leq j$ and $k \leq l$, and $[i j] \leq[k l]$ which means $i \leq k$ or $i=k$ but $j \leq l$ (lexicographic order). The remaining entries retrieve from consideration of index symmetry. The reader will note that the additional symmetry $\left\langle\mathbf{T}^{2}\right\rangle_{i j k l}=\left\langle\mathbf{T}^{2}\right\rangle_{k l i j}$ appears due to processing.

4.1.1.c. When the processing symmetry is "wire-like", the symmetry group is

$$
P=D_{\infty h}=\left\{\left[\begin{array}{ccc}
\cos \phi & \sin \phi & 0 \\
-\sin \varphi & \cos \phi & 0 \\
0 & 0 & \pm 1
\end{array}\right],\left[\begin{array}{ccc}
\cos \phi & \sin \phi & 0 \\
\sin \phi & -\cos \phi & 0 \\
0 & 0 & \pm 1
\end{array}\right]\right\}
$$

It follows that when $M>2$ the answer obtained is identical to that given in 4.1.1.a. When $M=2$, the answer obtained is identical to that given in 4.1.1.b. When $M=1$ the residence space of $\langle\mathbf{T}\rangle$ is $W\left(2, S_{2}, I\right)^{D_{\infty h}}$, which is twodimensional. The elements of this space are tensors of diagonal form with $\langle\mathbf{T}\rangle_{11}=\langle\mathbf{T}\rangle_{22}$; the independent parameters are $\langle\mathbf{T}\rangle_{11}$ and $\langle\mathbf{T}\rangle_{33}$.

4.1.2. Consider symmetric material tensors $T$ of order 2 with no crystal symmetry, and assuming no orientation correlation between distinct grains according to the model described in 2.3. Then the space $U(k, \Sigma, \Gamma, M)$ defined above is just $S^{M} \mathscr{T}(2, \Sigma)$ and it has dimension

$$
\left(\begin{array}{c}
M+5 \\
M
\end{array}\right)=\frac{(M+1)(M+2)(M+3)(M+4)(M+5)}{120} .
$$

4.1.2.a. If there is no processing symmetry (i.e. $P$ is trivial) then the residence space of $\left\langle\mathbf{T}^{M}\right\rangle^{0}$ is $U(2, \Sigma, \Gamma, M)$, and the independent parameters are $\left\langle\mathbf{T}^{M}\right\rangle_{i_{1} j_{1} \cdots i_{M} j_{M}}^{0}$ with $i_{n} \leq j_{n}, \quad 1 \leq n \leq M$, and $\left[i_{n} j_{n}\right] \leq\left[i_{n+1} j_{n+1}\right]$. The remaining components are retrieved by symmetries.

4.1.2.b. If $P$ is the orthotropic symmetry group $D_{2 h}$, the residence space of $\left\langle\mathbf{T}^{M}\right\rangle^{\mathbf{0}}$ is $\left(\boldsymbol{S}^{M} \mathscr{T}(2, \Sigma)\right)^{D_{2 h}}$. The independent parameters are now $\left\langle\mathbf{T}^{M}\right\rangle_{i_{1} j_{1} \cdots i_{M j}}^{\mathbf{0}}$ with indices satisfying the same conditions as enumerated in 4.1.2.a, plus an additional 
condition: there must be an even number of 1's, an even number of 2's, and an even number of 3's among the indices, otherwise the corresponding component is zero. This condition is equivalent to $D_{2 h}$-invariance. The remaining components are retrieved by symmetries.

An elementary calculation shows that the dimension of the residence space is 9 when $M=2$ and 20 when $M=3$. The list of index sets of independent components for $M=2$ is (1111), (1122), (1133), (1212), (1313), (2222), (2233), (2323), and (3333).

4.1.2.c. When $P$ is the "wire" symmetry group, $D_{\infty \text { h }}$, the residence space of $\left\langle\mathbf{T}^{M}\right\rangle^{0}$ is just $\left(S^{M} \mathscr{T}(2, \Sigma)\right)^{D_{\infty h}}$. When $M=2$ we have the following decomposition: $S^{2} \mathscr{T}(2, \Sigma)=2 M_{1}^{+} \oplus 2 M_{5}^{+} \oplus M_{9}^{+}$. The group $D_{\infty h}$ has a unique invariant (to within a scalar multiplicative factor) in every $M_{2 l+1}^{+}$. It follows that the residence space is 5-dimensional. The independent parameters of $\left\langle\mathbf{T}^{2}\right\rangle^{0}$ (assuming that the wire axis is the 3-axis) are the coefficients of the decomposition

$$
\begin{aligned}
\left\langle\mathrm{T}^{2}\right\rangle_{i j m n}^{0}= & \alpha_{1} \delta_{i j} \delta_{m n}+\alpha_{2}\left(\delta_{i m} \delta_{j n}+\delta_{i n} \delta_{j m}\right) \\
& +\alpha_{3}\left(v_{i j} \delta_{m n}+v_{m n} \delta_{i j}\right)+\alpha_{4} v_{i j} v_{m n}+\alpha_{5} D_{i j m n},
\end{aligned}
$$

where $v_{33}=1$ (all other $v_{i j}=0$ ) and $D_{i j m n}$ is a fourth-order symmetric and traceless tensor invariant under rotations about the 3-axis. The entries of $D$ are $D_{1111}=D_{2222}=3, D_{3333}=8, D_{1313}=D_{2323}=D_{1133}=D_{2233}=-4, D_{1212}=D_{1122}=1$, and all other entries are zero. The coefficients of the decomposition may be expressed in terms of $\left\langle\mathbf{T}^{2}\right\rangle^{0}(=\mathbf{Z})$ in the following manner: $\alpha_{1}=\left(4 Z_{j j 11}+2 Z_{j 333}-\right.$ $\left.2 Z_{j j 33}-3 Z_{j 1 j 1}\right) / 5, \quad \alpha_{2}=\left(Z_{j j 33}-Z_{j 3 j 3}+4 Z_{j 1 j 1}-2 Z_{j j 11}\right) / 10, \quad \alpha_{3}=Z_{1 j 1 j}+Z_{j j 33}-$ $Z_{j j 11}-Z_{j 3 j 3}, \quad \alpha_{4}=2 Z_{j j 11}-2 Z_{j j 33}+3 Z_{j 3 j 3}-3 Z_{j 1 j 1}, \quad \alpha_{5}=Z_{1212}-\alpha_{2}$. (In these expressions repeated $j$ indices imply summation from 1 to 3 .)

All of these results hold for any crystal symmetry group $\Gamma$ such that $M_{5}^{+}$is not empty, except the case $M \geq 3$ under the no-correlation assumptions associated with the model microstructure; in this case a further reduction occurs in the number of independent parameters. The dihedral groups, for instance, retain these properties. When $M_{5}^{+}$is empty with respect to $\Gamma$ then $\mathscr{T}(2, \Sigma)$ is the one-dimensional space of scalar tensors, therefore all correlation tensors will be scalars. This occurs, for example, when $\Gamma=O_{h}$, the symmetry group of the cube.

\subsection{Examples from Representations of Material Tensors of Order Four, Possessing No Crystal Symmetry}

4.2.1. Consider tensors of order four with index symmetries $T_{i j m n}=T_{j i m n}=$ $T_{i j n m}=T_{m n i j}$. Material tensors possessing such symmetry have extensive application in the infinitesimal linear elastic theory. Thus $k=4$, and the index symmetry group $\Sigma$ is an eight element subgroup in $S_{4}$ (the group of permutations of four items). It is well known that the space of these tensors, $\mathscr{T}(4, \Sigma)$, is 21dimensional. As an $O(3)$-representation it decomposes into $\mathscr{T}(4, \Sigma)=2 M_{1}^{+} \oplus$ $2 M_{5}^{+} \oplus M_{9}^{+}$.

4.2.1.a. When there is no processing symmetry the residence space of $\left\langle\mathbf{T}^{M}\right\rangle$ is just $\mathscr{T}(4, \Sigma)^{\otimes M}$ with dimension $21^{M}$. The independent parameters are $\left\langle\mathbf{T}^{M}\right\rangle_{i_{1} j_{1} m_{1} n_{1} \cdots i_{M} j_{M} m_{M} n_{M}}$, where $i_{p} \leq j_{p}, m_{p} \leq n_{p},\left[i_{p} j_{p}\right] \leq\left[m_{p} n_{p}\right]$ and $1 \leq p \leq M$. The remaining entries retrieve from the symmetry. 
4.2.1.b. Consider the case where the processing symmetry is the orthotropic group $P=D_{2 h}$. When $M>2$ the answers are identical to those just cited in 4.2.1.a. However, when $M=1$ the residence space of $\left\langle\mathbf{T}^{M}\right\rangle=\langle\mathbf{T}\rangle$ is just $(\mathscr{T}(4, \Sigma))^{D_{2 h}}$. Since $\left(M_{5}^{+}\right)^{D_{2 h}}$ is 2-dimensional, and $\left(M_{9}^{+}\right)^{D_{2 h}}$ is 3-dimensional, it follows that the residence space is 9-dimensional. The independent entries are those with indices (1111), (1122), (1133), (1313), (1212), (2233), (2222), (2323), and (3333). The remainder are retrieved from indicial symmetry, taking into account that any particular component is zero unless each of the numbers 1,2 , and 3 occur in the set of indices an even number of times.

When $M=2$ the residence space of $\left\langle T^{M}\right\rangle=\left\langle T^{2}\right\rangle$ is $S^{2} \mathscr{T}(4, \Sigma)$ which is 231-dimensional. The independent parameters are $\left\langle T^{2}\right\rangle_{i_{1} j_{1} m_{1} n_{1} i_{2} j_{2} m_{2} n_{2}}$ such that the indices have $i_{s} \leq j_{s}, m_{s} \leq n_{s},\left[i_{s} j_{s}\right] \leq\left[m_{s} n_{s}\right]$ for $s=1,2$, and $\left[i_{1} j_{1} m_{1} n_{1}\right] \leq$ $\left[i_{2} j_{2} m_{2} n_{2}\right]$ lexicographically. The remaining entries retrieve from the indicial symmetries $i j \rightarrow j i, m n \rightarrow n m, i j m n \rightarrow m n i j$ and the additional index symmetry $i_{1} j_{1} m_{1} n_{1} i_{2} j_{2} m_{2} n_{2} \rightarrow i_{2} j_{2} m_{2} n_{2} i_{1} j_{1} m_{1} n_{1}$ which is induced by the processing symmetry.

4.2.1.c. Now consider wire-like processing symmetry, $P=D_{\infty h}$. Again we find that when $M>2$ the results are identical to those described in 4.2.1.a. When $M=2$ the results described in 4.2.1.b apply. When $M=1$ the residence space of $\left\langle\mathbf{T}^{M}\right\rangle=\langle\mathbf{T}\rangle$ is $(\mathscr{T}(4, \Sigma))^{D_{\infty h}}$ which is 5-dimensional. The description of independent parameters coincides with those described in 4.1.2.c for the second-order tensor with $M=2$. The reason for this coincidence is clear; fourth-order tensors with index symmetry characteristic of elastic properties lie in $S^{2} \mathscr{T}\left(2, \Sigma_{2}\right)$ where $\mathscr{T}\left(2, \Sigma_{2}\right)$ is the space of symmetric tensors of order 2 .

4.2.2. Consider the case where no symmetry of the crystal lattice is present, as before, but in addition lattice orientation between grains or crystallites is not present according to the model microstructure defined in 2.3. For this case $U(4, \Sigma, M, \Gamma)=S^{M} \mathscr{T}(4, \Sigma)$ which has dimension $\left({ }_{M}^{M+20}\right)$.

4.2.2.a. If $P$ is trivial, the residence space of $\left\langle\mathbf{T}^{M}\right\rangle^{0}$ is exactly $U(4, \Sigma, M, \Gamma)$, and the independent components are $\left\langle\mathbf{T}^{M}\right\rangle_{i_{1} j_{1} m_{1} n_{1} \cdots i_{M} j_{M} m_{M} n_{M}}$ where the following conditions hold:

and

$$
i_{s} \leq j_{s}, m_{s} \leq n_{s},\left[i_{s} j_{s}\right] \leq\left[m_{s} n_{s}\right] \text { for } s=1, \ldots, M
$$

$$
\left[i_{s} j_{s} m_{s} n_{s}\right] \leq\left[i_{s+1} j_{s+1} m_{s+1} n_{s+1}\right] \text { for } 1 \leq s \leq M-1 .
$$

The remaining components are retrieved by symmetry.

4.2.2.b. Now consider the case of orthotropic processing symmetry, $P=D_{2 h}$. The residence space of $\left\langle T^{M}\right\rangle^{0}$ is $U(4, \Sigma, M, \Gamma)^{D_{2 h}}$. The independent components of $\left\langle\mathbf{T}^{M}\right\rangle^{0}$ are identical to those described in 4.2.2.a with an additional index condition: there must be an even number of 1's, 2's and 3's among $i_{s} j_{s} m_{s} n_{s}$ for $1 \leq s \leq M$. The dimension of the residence space (i.e. the number of independent components) is 75 if $M=2$ and 499 if $M=3$.

4.2.2.c. If $P$ is the wire-like symmetry group $P=D_{\infty h}$, the residence space $U(4, \Sigma, M, \Gamma)^{D_{\alpha h}}$ of $\left\langle T^{M}\right\rangle^{0}$ has dimension 35 if $M=2$ and 207 if $M=3$. 
We shall require operators $E$ and $F$ acting on tensors. Their action is defined as

$$
\begin{aligned}
& E \cdot R_{j_{1} \cdots j_{q}}=\sum_{s=1}^{q} \sum_{p=1}^{3} R_{j_{1} \cdots j_{s-1} p j_{s+1} \cdots j_{q}} \cdot e_{j_{s} p}, \\
& F \cdot R_{j_{1} \cdots j_{q}}=\sum_{s=1}^{q} \sum_{p=1}^{3} R_{j_{1} \cdots j_{s-1} p j_{s+1} \cdots j_{q}} \cdot f_{j_{s} p},
\end{aligned}
$$

where

$$
e=\left[\begin{array}{ccc}
0 & 0 & -1 \\
0 & 0 & i \\
1 & -i & 0
\end{array}\right], \quad f=\left[\begin{array}{ccc}
0 & 0 & -1 \\
0 & 0 & -i \\
1 & i & 0
\end{array}\right], \quad i=\sqrt{-1}
$$

For a tensor $\mathbf{R}=\mathbf{R}^{(0)}$, we denote $E^{s} \cdot \mathbf{R}$ by $\mathbf{R}^{(s)}$ and $F^{s} \cdot \mathbf{R}$ by $\mathbf{R}^{(-s)}$. Note that $\mathbf{R}^{(s)}$ and $\mathbf{R}^{(-s)}$ will have complex components. They are used to construct a basis for the residence space of $\left\langle\mathbf{T}^{2}\right\rangle^{0}$. Even though $\mathbf{R}^{(s)}$ and $\mathbf{R}^{(-s)}$ are complex-valued, the basis is real. We require the following definitions

$$
\begin{gathered}
Q_{i j m n}^{(1,0)}=\delta_{i j} \delta_{m n}, \quad Q_{i j m n}^{(2,0)}=\delta_{i m} \delta_{j n}+\delta_{i n} \delta_{j m}, \\
Q_{i j m n}^{(3, s)}=\left(\delta_{i j} \mu_{m n}+\delta_{m n} \mu_{i j}\right)^{(s)}, \quad-2 \leq s \leq 2, \\
Q_{i j m n}^{(4, s)}=\left(\mu_{i j} \mu_{m n}\right)^{(s)}, \quad-2 \leq s \leq 2, \\
Q_{i j m n}^{(5, s)}=D_{i j m n}^{(s)}, \quad-4 \leq s \leq 4,
\end{gathered}
$$

where $\mathrm{D}$ has been previously defined in 4.1.2.c and $\mu$ is the symmetric and traceless second order tensor with matrix of coefficients

$$
\mu_{i j}=\left[\begin{array}{rrr}
1 & 0 & 0 \\
0 & 1 & 0 \\
0 & 0 & -2
\end{array}\right]
$$

It can be shown that the following elements form an orthogonal basis of the residence space for $\left\langle\mathbf{T}^{2}\right\rangle^{0}$ :

$$
\begin{gathered}
\mathbf{P}^{q_{1}, q_{2}, 0}={ }_{\mathrm{def}} \mathbf{Q}^{\left(q_{1}, 0\right)} \otimes \mathbf{Q}^{\left(q_{2}, 0\right)}+\mathbf{Q}^{\left(q_{2}, 0\right)} \otimes \mathbf{Q}^{\left(q_{1}, 0\right)} \\
1 \leq q_{1} \leq q_{2} \leq 5, \quad(15 \text { elements }), \\
\mathbf{P}^{q_{1}, q_{2}, s}={ }_{\mathrm{def}} \mathbf{Q}^{\left(q_{1}, s\right)} \otimes \mathbf{Q}^{\left(q_{2},-s\right)}+\mathbf{Q}^{\left(q_{1},-s\right)} \otimes \mathbf{Q}^{\left(q_{2}, s\right)} \\
+\mathbf{Q}^{\left(q_{2}, s\right)} \otimes \mathbf{Q}^{\left(q_{1},-s\right)}+\mathbf{Q}^{\left(q_{2},-s\right)} \otimes \mathbf{Q}^{\left(q_{1}, s\right)} \\
1 \leq s \leq 4,1 \leq q_{1} \leq q_{2} \leq r(s), \\
r(s)=3 \text { if } s=1 \text { or } 2, r(s)=1 \text { if } s=3 \text { or } 4, \quad(20 \text { elements }) .
\end{gathered}
$$

This follows from the fact that the tensors $\mathbf{Q}^{(q, s)}$ are orthogonal to one another. The independent parameters of $\left\langle\mathbf{T}^{2}\right\rangle^{0}$ are the coefficients of the decomposition

$$
\left\langle\mathbf{T}^{2}\right\rangle=\sum_{q_{1}, q_{2}, s} \alpha_{q_{1} q_{2}}^{s} \mathbf{P}^{q_{1}, q_{2}, s} .
$$

Since this decomposition is orthogonal, the coefficients $\alpha_{q_{1} q_{2}}^{s}$ can be retrieved 
from $\left\langle\mathbf{T}^{2}\right\rangle^{0}$ as follows:

$$
\alpha_{q_{1} q_{2}}^{s}=\frac{\left\langle T^{2}\right\rangle^{0} \cdot \mathbf{P}^{q_{1}, q_{2}, s}}{\mathbf{P}^{q_{1}, q_{2}, s} \cdot \mathbf{P}^{q_{1}, q_{2}, s}}
$$

where $\cdot$ indicates contraction of tensors.

\subsection{Examples from Representation of Material Tensors of Order Four, Possessing Cubic Crystal Symmetry}

If neither $M_{5}^{+}$nor $M_{9}^{+}$is empty with respect to the crystal symmetry group $\Gamma$, the results for correlation tensors possessing indicial symmetry typical of elasticity tensors (as described in 4.2.1) are identical with the results already presented for the case when $\Gamma$ is trivial, except in the case $M \geq 2$ when a no-correlation (model microstructure) assumption is made (in this case there will be a reduction in the number of independent parameters). Such is the case, to cite an example, when $\Gamma$ is the dihedral (trigonal or hexagonal) symmetry group. This is not the case when $\Gamma$ is the symmetry group of the cube, $\Gamma=O_{h}$, which contains 48 elements. This interesting and technologically important case is now considered separately.

4.3.1. In this case $W\left(4, \Sigma, O_{h}\right)=2 M_{1}^{+} \oplus M_{9}^{+}$. The two $M_{5}^{+}$components originally present when the lattice symmetry group was trivial, are now absent in the decomposition because they contain no invariants of $O_{h}$. The dimension is now 11.

4.3.1.a. When no processing symmetry is present (i.e. $P$ is the trivial group) the residence space of $\left\langle\mathbf{T}^{M}\right\rangle$ is $W\left(4, \Sigma, O_{h}\right)^{\otimes M}$ which is $11^{M}$ dimensional.

First consider the case when $M=1$. Recall that $\mathbf{D} \in M_{9}^{+}$. Generate a realvalued orthogonal basis of $W\left(4, \Sigma, O_{h}\right)$ as follows:

$$
\begin{gathered}
\mathbf{P}_{i j m n}^{1}=\delta_{i j} \delta_{m n} ; \quad \mathbf{P}_{i j m n}^{2}=\delta_{i m} \delta_{j n}+\delta_{i n} \delta_{j m} ; \\
\mathbf{P}_{i j m n}^{q}=\left(D_{i j m n}^{(7-q)}-D_{i j m n}^{(q-7)}\right) / i, \quad 3 \leq q \leq 6 ; \quad \mathbf{P}_{i j m n}^{7}=D_{i j m n} ; \\
\mathbf{P}_{i j m n}^{q}=D_{i j m n}^{(q-7)}+D_{i j m n}^{(7-q)}, \quad 8 \leq q \leq 11 .
\end{gathered}
$$

The independent parameters of $\langle T\rangle$ are the coefficients $\alpha_{q}(1 \leq q \leq 11)$ of the decomposition

$$
\langle\mathbf{T}\rangle=\sum_{q=1}^{11} \alpha_{q} \mathbf{P}^{q},
$$

and because of orthogonality $\alpha_{q}$ can be retrieved from the expression

$$
\alpha_{q}=\langle\mathbf{T}\rangle \cdot \mathbf{P}^{q} / \mathbf{P}^{q} \cdot \mathbf{P}^{q}, \quad 1 \leq q \leq 11 .
$$

When $M \geq 2$, the independent parameters are $\alpha_{q_{1} \cdots q_{M}}$; i.e. the coefficients of the decomposition

$$
\left\langle\mathbf{T}^{M}\right\rangle=\sum_{q_{1}, \ldots, q_{M}=1}^{11} \alpha_{q_{1}, \ldots, q_{M}} \mathbf{P}^{q_{1}, \ldots, q_{M}},
$$


where $\mathbf{P}^{q_{1}, \ldots, q_{M}}=\mathbf{p}^{q_{1}} \otimes \cdots \otimes \mathbf{p}^{q_{M}}$. The coefficients $\alpha_{q_{1}, \ldots, q_{M}}$ are retrieved from an expression exactly analogous to that for the case $M=1$.

4.3.1.b. When the processing symmetry is orthotropic (i.e. $P=D_{2 h}$ ), when $M \geq 3$ the residence space is exactly the same as in 4.3.1.a. When $M=1$, the dimension of the residence space, $W\left(4, \Sigma, O_{h}\right)^{D_{2 h}}$, is 5 since there are three independent $D_{2 h}$ invariants in $M_{9}^{+}$. The decomposition of 4.3.1.a applies here, but we will have the restriction that $\alpha_{q}=0$ when $q=3,4,5,6,8$, and 10. The independent parameters are thus $\alpha_{1}, \alpha_{2}, \alpha_{7}, \alpha_{9}$, and $\alpha_{11}$.

When $M=2$ the residence space is $S^{2} W\left(4, \Sigma, O_{h}\right)$, which has dimension 66 . The independent parameters are the coefficients $\alpha_{q_{1} q_{2}}$ of the decomposition cited in 4.3.1.a with the added restriction that $q_{1} \leq q_{2}$, since now $\alpha_{q_{1} q_{2}}=\alpha_{q_{2} q_{1}}$.

4.3.1.c. Now consider the case of wire-like symmetry $\left(P=D_{\infty \mathrm{h}}\right)$. In this case we need only consider the case where $M=1$; for $M=2$ all is the same as in 4.3.1.b, and when $M \geq 3$ the results are identical to those presented in 4.3.1.a.

From $M=1$ the residence space is 3-dimensional since there is only one $D_{\infty h}$-invariant in $M_{9}^{+}$. The decomposition reduces to

$$
\langle T\rangle_{i j m n}=\alpha_{1} \delta_{i j} \delta_{m n}+\alpha_{2}\left(\delta_{i m} \delta_{j n}+\delta_{i n} \delta_{j m}\right)+\alpha_{7} D_{i j m n}
$$

The coefficients are shown to be

$$
\begin{gathered}
\alpha_{1}=\left(\langle T\rangle_{j j 11}-\langle T\rangle_{j 1 j 1}\right) / 5, \quad \alpha_{2}=\left(3\langle T\rangle_{j 1 j 1}-\langle T\rangle_{j j 11}\right) / 10, \\
\alpha_{7}=\langle T\rangle_{1212}-\alpha_{2} .
\end{gathered}
$$

4.3.2. Consider next the model microstructure described in 2.3 (incorporating a no correlation assumption). We will treat only the case $M=2$ for simplicity. The space $U\left(4, \Sigma, O_{h}, 2\right)$ has dimension 61 , and as a representation of $O(3)$ it decomposes as

$$
M U=4 M_{1}^{+} \oplus 3 M_{9}^{+} \oplus M_{13}^{+} \oplus M_{17}^{+} .
$$

4.3.2.a. When no processing symmetry is present the residence space of $\left\langle\mathbf{T}^{2}\right\rangle^{0}$ is $U\left(4, \Sigma, O_{h}, 2\right)$. The parameters characterizing $\left\langle\mathbf{T}^{2}\right\rangle^{0}$, are $\alpha_{q_{1} q_{2}}, q_{1} \leq q_{2}$, and the manner of obtaining them is identical to that described in 4.3.1.a. However, there are 66 such parameters, so there will be five linear relations between them. These relations appear because we have eliminated the empty term $M_{5}^{+}$from the decomposition of $S^{2} W$, reducing the dimension from 66 to 61 . They can be written down without too much trouble, but we will not do so here.

4.3.2.b. When orthotropic processing symmetry is present $\left(P=D_{2 h}\right)$ the dimension of the residence space $U\left(4, \Sigma, O_{h}, 2\right)^{D_{2 h}}$ is 22 . The parameters of $\left\langle T^{2}\right\rangle^{0}$ in this case are:

$$
\begin{array}{ll}
\alpha_{q_{1} q_{2}}, & \text { when } q_{1}, q_{2}=1,2,7,9,11, q_{1} \leq q_{2}(15 \text { parameters }) \\
\alpha_{q_{1} q_{2}}, & \text { when } q_{1}, q_{2}=3,5, q_{1} \leq q_{2}(3 \text { parameters }) \\
\alpha_{q_{1} q_{2}}, & \text { when } q_{1}, q_{2}=4,6, q_{1} \leq q_{2}(3 \text { parameters }) \\
\alpha_{q_{1} q_{2}}, & \text { when } q_{1}, q_{2}=8,10, q_{1} \leq q_{2}(3 \text { parameters })
\end{array}
$$


We note that if the pair $\left(q_{1}, q_{2}\right)$, with $q_{1} \leq q_{2}$, satisfies none of these conditions, then $\alpha_{q_{1} q_{2}}=0$. The procedure for determining the $\alpha_{q_{1} q_{2}}$ coefficients is as described in 4.3.1.a. Note that since we have described 24 parameters, they will satisfy two linear relations. As before, they arise from the eliminated empty term $M_{5}^{+}$.

4.3.2.c. For wire-like processing symmetry $\left(P=D_{\infty h}\right)$ the dimension of the residence space $U\left(4, \Sigma, O_{h}, 2\right)^{D_{1} h}$ is 9 . Select a complex orthonormal basis of $W\left(4, \Sigma, O_{h}\right)$ with

$H_{i j m n}^{1}=\delta_{i j} \delta_{m n} ; \quad H_{i j m n}^{2}=\delta_{i m} \delta_{j n}+\delta_{i n} \delta_{j m} ; \quad H_{i j m n}^{q}=D_{i j m n}^{(q-7)}, \quad 3<q \leq 11$.

The parameters of $\left\langle\mathbf{T}^{2}\right\rangle^{0}$ are the coefficients of the decomposition

$$
\left\langle\mathbf{T}^{2}\right\rangle^{\mathbf{0}}=\sum_{q_{1}, q_{2}} \alpha_{q_{1}, q_{2}} \mathbf{H}^{q_{1}, q_{2}},
$$

where

$$
\mathbf{H}^{q_{1}, q_{2}}={ }_{\text {def }} \mathbf{H}^{q_{1}} \otimes \mathbf{H}^{q_{2}},
$$

and pairs $\left(q_{1}, q_{2}\right)$ are among the following (up to order): $(1,1),(1,2),(2,2)$, $(1,7),(2,7),(7,7),(6,8),(5,9),(4,10)$, and $(3,11)$. Because of symmetry $\alpha_{q_{1} q_{2}}=\alpha_{q_{2} q_{1}}$. These coefficients are retrieved in the familiar manner from $\left\langle\mathbf{T}^{2}\right\rangle^{0}$. Since there are 10 such coefficients, they will satisfy one linear relation, again originating from the empty term $M_{5}^{+}$which has been eliminated.

\section{SUMMARY}

In the development described above, we have considered the $n$-point correlation tensors as a particular class of quantitative representation of polycrystalline microstructure. Although in general we must assume that the spatial coherence of lattice orientation extends beyond the scale of the crystallites themselves (i.e. into 1st and 2nd nearest neighbors, etc.), there is mounting experimental evidence that for some polycrystals it is reasonable to assume that a coherence persists only over distances associated with finite crystallite size. For such a "model" microstructure there obtains substantial simplification in the point statistics (cf. Eqs. (20) and (21) in the text) and in the residence space of the corresponding correlation tensors.

The central problem of this paper has been to show the action of various aspects of symmetry on the residence spaces of the $n$-point correlation tensors. Beginning with a consideration of the action of $O(3)$ on the space of tensors of rank $k, V(k)$, we have illustrated the reductions which occur in representation due to the presence of indicial symmetry in the coefficients of the tensor, the symmetries of the crystal lattice, and statistical symmetries occurring due to processing. The group-theoretical framework for reducing the residence space, from its general form to the smallest possible (least dimension) form, has been described in general, and in several specific examples.

Knowing the residence space for any particular correlation tensor can substantially reduce the computational labor required in forming properties estimates. In fact, the dimension of some spaces of interest would be completely out of the 
realm of current computational possibility when the reductions due to symmetry are not considered. There is another interest for such efforts in symmetrization, however, which seems equally important. This can be best described as a clarification of the physical nature of the models which incorporate the correlation tensors. Very often finding the smallest possible residence space can substantially clarify physical theory.

\section{Acknowledgements}

The authors wish to acknowledge that this work was sponsored through a Materials Research Groups Award by the National Science Foundation. Helpful discussions with Professor E. Turan Onat, and Alexander A. Kirillov, Jr. are gratefully acknowledged.

\section{References}

Adams, B. L., Morris, P. R., Wang, T. T., Willden, K. S. and Wright, S. I. (1987). Acta metall. 35, 2935.

Adams, B. L., Wang, T. T. and Morris, P. R. (1988). In Proc. 8th Int. Conf. Textures of Materials, p. 135, eds. J. Kallend and G. Göttstein, TMS-AIME, Philadelphia.

Adams, B. L. and Onat, E. T. (1991). In Proc. 9th Int. Conf. Textures of Materials, p., guest eds. R. Pennelle and C. Esling, Textures and Microstructures 14-18.

Adams, B. L. and Field, D. P. (1991). Acta metall. mater. 39, 2405.

Adams, B. L., Guidi, M., Boehler, J. P. and Onat, E. T. (1992). J. Mech. Phys. Solids, in press.

Beran, M. and Molyneux, J. (1966) Q. Appl. Math. 24, 107.

Broecker, T. and tom Dieck, T. (1985). Representation of Compact Lie Groups, Springer, New York.

Gel'fand, I. M., Minlos, R. A. and Shapiro, A. Ya. (1963). Representations of the Rotation and Lorentz Groups and their Applications, Pergamon, Oxford.

Guidi, M., Adams, B. L. and Onat, E. T. (1992). Textures and Microstructures, in press.

Hashin, Z. and Shtrikman, S. (1962). J. Appl. Phys. 33, 3125.

Kunze, K., Wright, S. I. and Adams, B. L. (1992). Textures and Microstructures, submitted.

Kröner, E. (1986). In Modelling Small Deformations of Polycrystals, p. 229, eds. J. Gittus and J. Zarka, Elsevier Applied Science Publishers, New York.

McCoy, J. J. (1981). In Mechanics Today 6, ed. S. Nemat-Nasser, p. 1.

Ponte Casteñada, P. (1991). J. Mech. Phys. Solids 39, 45.

Talbot, D. R. S. and Willis, J. R. (1985). IMA J. appl. Math. 35, 39.

Wang, T. T., Morris, P. R. and Adams, B. L. (1990). Metall. Trans. 21A, 2265.

Willis, J. R. (1983). J. appl. Mech. 50, 1202.

Willis, J. R. (1981). In Advances in Applied Mechanics 21, p. 2, ed. C. S. Yih, Academic Press, New York.

Wright, S. I. and Adams, B. L. (1992). Metall. Trans. 23A, 759. 